\title{
LARGE SIALOLITH IN SUBMANDIBULAR GLAND PARENCHYMA - A CASE REPORT
}

\author{
Dr Kshitija Bhakte ${ }^{1}$, Dr Ramhari Sathawane ${ }^{2}$, Dr Rakhi Chandak ${ }^{3}$, Dr Ashish Lanjekar ${ }^{3}$, Dr Romita \\ Gaikwad $^{4}$, Dr Gunjan Moon ${ }^{1}$, Dr Lajri Bagde ${ }^{1}$ \\ 1- Postgraduate Student , 2- Professor and Guide, 3- Reader, 4- Sr Lecturer, Department Of Oral Medicine and \\ Radiology, Swargiya Dadasaheb Kalmegh Smruti Dental College and Hospital, Nagpur
}

\begin{abstract}
Sialolith is the most common obstructive pathology of the salivary gland. Submandidular glands are most commonly affected in its parenchyma. This case report describes a patient presenting with an unusually large submandibular gland sialolith, where sialography was performed locating the lesion. Etiology, diagnosis and various treatment modalities available for management of salivary gland calculi depending on their site and size are also discussed in this case report.
\end{abstract}

KEYWORDS: Sialolith, Submandidular gland, Sialography

\section{INTRODUCTION}

Salivary glands are the saliva secreting glands through duct in the body, that keeps the oral cavity moist and helps in reduction in pathology due to reduced flow due to its flushing action. Sialolithiasis is an obstructive and most common disease of salivary glands. Males are commonly affected as much twice than females. Sialolithiasis occurs mainly in major salivary glands, perhaps minor salivary glands may also be affected. It accounts for $50 \%$ of diseases of salivary glands and is thus the most common cause of acute and chronic infections.

More than $80 \%$ occur in the submandibular gland or its duct, $6 \%$ in the parotid gland and $2 \%$ in the sublingual gland or minor salivary glands ${ }^{1}$. Forty per cent of parotid and $20 \%$ of submandibular stones are not radiopaque and sialography is required to locate them ${ }^{2}$. Sialolith is usually unilateral and are not a cause of dry mouth. Clinically they are round or ovoid, rough or smooth and of a yellowish colour. They consist of mainly calcium phosphate alongwith smaller amounts of carbonates in the form of hydroxyapatite, with smaller amounts of magnesium, potassium and ammonia. Submandibular stones are $82 \%$ inorganic and $18 \%$ organic material whereas parotid stones are composed of $49 \%$ inorganic and $51 \%$ organic material $^{3}$. The organic material is composed of various carbohydrates and amino acids. Bacterial elements have not been identified at the core of a sialolith.

\section{CASE REPORT}

A 35-year-old male patient reported to the Department of Oral Medicine and Maxillofacial Radiology with the chief complaint of pain and intermittent swelling while eating food in lower left back region of jaw since 1 month. Extra-oral examination revealed an enlarged and tender left submandibular gland. He was advised an OPG. OPG revealed a single, ovoid, irregular, homogenous radiopacity with smooth margins near the angle of mandible and in approximation with the inner cortex of the mandible. The size was about 
$4 \times 3 \mathrm{~cm}$ approximately. (Fig 1)

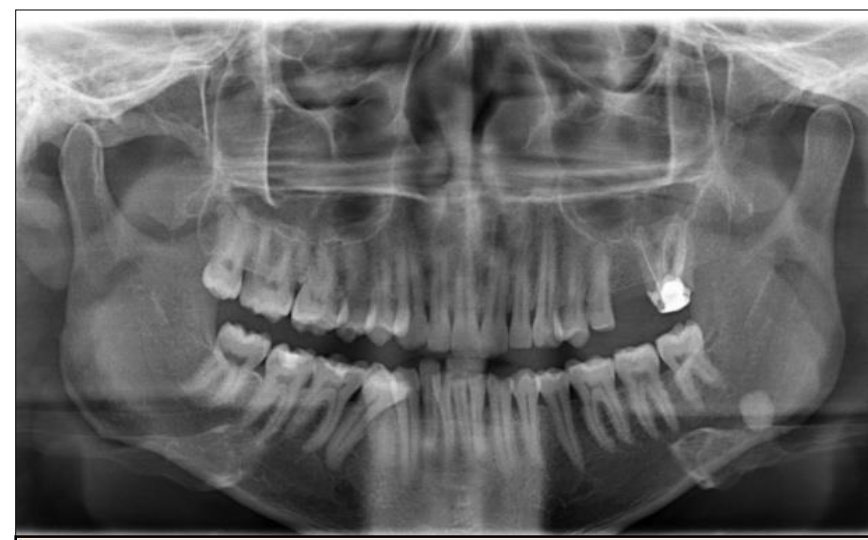

Figure 1: OPG showing large sialolith in left submandibular salivary gland just above inferior border of mandible.

A final diagnosis of left large sialolith in gland parenchyma near proximal end of Wharton's duct was made. Patient was prescribed antibiotics for 5 days and instructed to have increased intake of water. He was advised left submandibular salivary gland sialography after 7 days.

The sialography was planned and under all safety precautions sialography was performed for left submandibular gland, where initially a periodontal probe end ball \& lacrimal probe was used to dilate the Wharton's duct. After sufficient dilatation the cannula was inserted into the duct and water based sialography dye $0.7-1.0 \mathrm{ml}$ was injected slowly into the duct/gland. The OPG was taken which demonstrated that the dye filled the proximal portion of the duct excluding the sialolith that typically gives appearance of the sialolith (Fig 2).

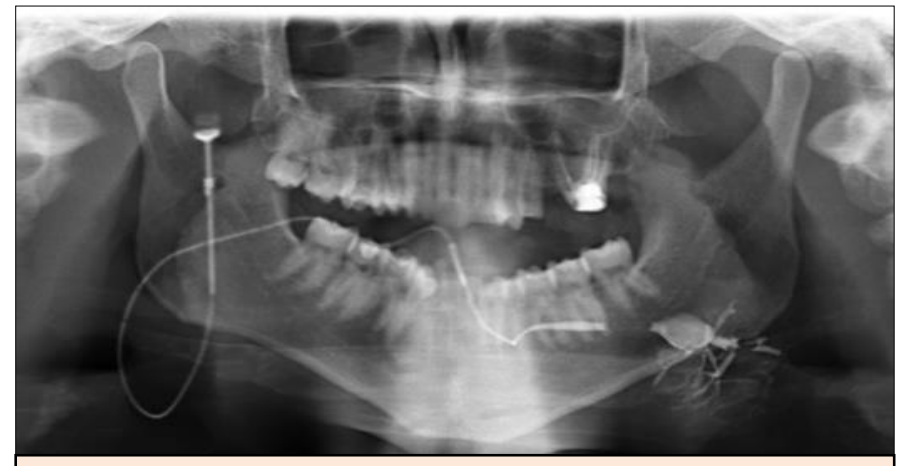

Figure 2: Sialography of Left submandibular salivary gland showing large calculus and glandular architecture.
The patient was given sialogogue to remove the injected dye. Then the patient was referred to Oral Surgery for sialolithotripsy. He was advised total removal of left salivary gland. He was not willing for surgical removal. Therefore, he was advised to continue conservative management i.e increased intake of water, use of sialologues, moist warm heat application and gland massage. alongwith regular follow ups.

\section{DISCUSSION}

The exact etiology and pathogenesis of salivary calculi is largely unknown. Genesis of calculi lies in the relative stagnation of calcium rich saliva. They are thought to occur as a result of deposition of calcium salts around an initial organic nidus consisting of altered salivary mucins, bacteria and desquamated epithelial cells. ${ }^{3}$ For stone formation it is likely that intermittent stasis produces a change in the mucoid element of saliva, which forms a gel. This gel produces the framework for deposition of salts and organic substances creating a stone.

Traditional theories suggest that the formation occurs in two phases: a central core and a layered periphery. The central core is formed by the precipitation of salts, which are bound by certain organic substances. The second phase consists of the layered deposition of organic and non organic material. Submandibular stones are thought to form around a nidus of mucous, whereas parotid stones are thought to form most often around a nidus of inflammatory cells or a foreign body. ${ }^{4}$

Another theory has proposed that an unknown metabolic phenomenon can increase the salivary bicarbonate content, which alters calcium phosphate solubility and leads to precipitation of calcium and phosphate ions. A retrograde theory for sialolithiasis has also been proposed. Other substances or bacteria within the oral cavity might migrate into the salivary ducts and become the nidus for further calcification. ${ }^{5}$

Submandibular sialolithiasis is more common as its saliva is (i) more alkaline, (ii) has an increased 
concentration of calcium and phosphate, and (iii) has a higher mucous content than saliva of the parotid and sublingual glands. In addition, the submandibular duct is longer and the gland has an antigravity flow. Stone formation is not associated with systemic abnormalities of calcium metabolism. ${ }^{6}$ Electrolytes and parathyroid hormone studies in patients with sialolithiasis have not shown abnormalities. Gout is the only systemic illness known to predispose to salivary stone formation, although in gout the stones are made predominantly of uric acid. ${ }^{7}$ Study by Hemminki $\mathrm{K}$ et al has suggested a link between sialolithiasis and nephrolithiasis, reporting an association in up to $10 \%$ of patients. ${ }^{8}$

Sialolithiasis typically causes pain and swelling of the involved salivary gland by obstructing the food related surge of salivary secretion. Calculi may cause stasis of saliva, leading to bacterial ascent into the parenchyma of the gland, and therefore infection, pain and swelling of the gland. Some may be asymptomatic until the stone passes forward and can be palpated in the duct or seen at the duct orifice. It may be possible that obstruction caused by large calculi is sometimes asymptomatic as obstruction is not complete and some saliva manages to seep through or around the calculus. Long term obstruction in the absence of infection can lead to atrophy of the gland with resultant lack of secretory function and ultimately fibrosis ${ }^{9}$.

Patients presenting with sialolithiasis may benefit from a trial of conservative management, especially if the stone is small. The patient must be well hydrated and the clinician must apply moist warm heat and gland massage, while sialogogues are used to promote saliva production and flush the stone out of the duct. With gland swelling and sialolithiasis, infection should be assumed and a penicillinase resistant anti staphylococcal antibiotic prescribed. Most stones will respond to such a regimen, combined with simple sialolithotomy when required. ${ }^{10}$ Almost half of the submandibular calculi lie in the distal third of the duct and are amenable to simple surgical release through an incision in the floor of the mouth, which is relatively simple to perform and not usually associated with complications.

If the stone is sufficiently forward it can be milked and manipulated through the duct orifice. This can be done with the aid of lacrimal probes and dilators to open the duct. Once open, the stone can be identified, milked forward, grasped and removed. The gland is then milked to remove any other debris in the more posterior portion of the duct. The duct may need opening to retrieve the stone. This involves a transoral approach where an incision is made directly onto the stone. In this way more posterior stones, $1-2 \mathrm{~cm}$ from the punctum, can be removed by cutting directly onto the stone in the longitudinal axis of the duct. Care is taken as the lingual nerve lies deep, but in close association with the submandibular duct posteriorly. Subsequently, the stone can be grasped and removed. No closure is done leaving the duct open for drainage. If the gland has been damaged by recurrent infection and fibrosis, or calculi have formed within the gland, it may require removal. ${ }^{11}$

Alternative methods of treatment have emerged such as the use of extracorporeal shock wave lithotripsy (ESWL) and more recently the use of endoscopic intracorporeal shockwave lithotripsy (EISWL), in which shockwaves are delivered directly to the surface of the stone lodged within the duct without damaging adjacent tissue (piezoelectric principle). ${ }^{12}$ Both extra and intracorporeal lithotripsy are gaining increasing importance in the treatment of salivary stone disease. In extracorporeal piezoelectric lithotripsy, the average size of fragments produced is about 0.7 $\mathrm{mm}$. Duct diameters are greater than $0.7 \mathrm{~mm}$ in general except for at the ostium. Therefore, fragments produced by ESWL would not be prohibited by duct diameters. ${ }^{13}$

Findings have also suggested that best results in salivary stone lithotripsy are achieved when the 
maximum size of stone fragments does not exceed $1.2 \mathrm{~mm}$. Extracorporeal salivary lithotripsy provides another therapeutic option that carries fewer risks than surgical removal of the affected gland, such as the risks of general anaesthetia, facial nerve damage, surgical scar, Frey's syndrome, and causes little discomfort to the patient whilst preserving the gland. ${ }^{14} \mathrm{~A}$ retrospective study of patients treated endoscopically from 1994 to 1999 showed a success rate of $83 \%$ with no severe complications. Endoscopy is a minimally invasive technique for removal of calculi from salivary glands as well as an excellent diagnostic procedure, as miniaturised endoscopes conforming to the physiological widths of the ducts are used to directly view and then deliver shock waves to the stones. ${ }^{15}$

\section{CONCLUSION}

There are various methods available for imaging of the salivary gland but sialography has been reported to be the most appropriate one, since it is the only method which demonstrates glandular architecture.

The management of salivary stones, depends on the gland affected and stone location. However, ESWL and EISWL offer alternatives to gland removal. Submandibular gland removal may be indicated following failure of lithotripsy or if the size of an intraglandular stone reaches $12 \mathrm{~mm}$ or more as the success of lithotripsy may be less than $20 \%$ in such cases.

Financial support and sponsorship: Nil.

Conflicts of interest: There are no conflicts of interest.

\section{REFERENCES}

1. Holden AM et al. Audit of minimally invasive surgery for submandibular sialolithiasis. British Journal of Oral and Maxillofacial Surgery, 2019 July (57), 582-586.

2. Costan VV, et al. Use of Cone-Beam Computed Tomography in Performing Submandibular
Sialolithotomy. J Oral Maxillofac Surg. 2019 Aug;77(8):1656-1660.

3. Arifa SP. Sialolithiasis of the Submandibular Gland: Case reports Cureus. 2019 Mar;11(3)17- 20.

4. Romero NJ et al . Sonolocation during submandibular sialolithotomy. The Laryngoscope. 2019 Feb 23,19-24.

5. Guenzel Tet al. Sialendoscopy plus laser lithotripsy in sialolithiasis of the submandibular gland in 64 patients: A simple and safe procedure 2019 Feb 11,61-65.

6. Kraaij $\mathrm{S}$ et al Biochemical composition of salivary stones in relation to stone-and patientrelated factors. Medicina oral, patologia oral y cirugia bucal. 2018 Sep;23(5):e540-546.

7. Blatt im et al Studies in sialolithiasis. II. Uric acid calculus of the parotid gland; report of a case. Ann Otol Rhinol Laryngol. 1958 Dec;67(4):102232 .

8. Hemminki $\mathrm{K}$ et al Familial risks in and between stone diseases: sialolithiasis, urolithiasis and cholelithiasis in the population of Sweden. BMC nephrology. 2018 Dec;19(1):158-162.

9. Aiyekomogbon JO et al. Submandibular sialolithiasis: The roles of radiology in its diagnosis and treatment. Annals of African medicine. 2018 Oct;17(4):221-225.

10. Fabie JE et al. Gland preserving surgery for salivary stones and the utility of sialendoscopes. Head \& neck. 2019 May;41(5):1320-7.

11. Marchul $\mathrm{F}$ et al Retrograde Theory in sialolithiasis formation. Archives of Otolaryngology- Head and Neck Surgery 2001. 127: 66-8.

12. Rauch $\mathrm{S}$ et al Disease of the salivary glands. In: Gorlin R J, Goldmann H M, eds. Thomas' Oral Pathology. St Loius, Mo: Mosby-Year Book Inc; 1970: 997-1003. 
13. Pollack C V et al. Sialolithisis: case studies and review. J Emergency Medicine 1990. 8: 561-565.

14. Pietz D Met al . Submandibular sialolithisis.

General Dentistry1987; 35: 494-496.

15. Rice $\mathrm{D}$ H. Advances in diagnosis and management of salivary gland diseases. West J Med 1984; 140: 148 . 